\title{
Degrees in oriented hypergraphs and Ramsey $p$-chromatic number
}

\author{
Yair Caro \\ Dept. of Mathematics and Physics \\ University of Haifa-Oranim \\ Tivon 36006, Israel \\ yacaro@kvgeva.org.il
}

\author{
Adriana Hansberg* \\ Dep. de Matemàtica Aplicada III \\ UPC Barcelona \\ 08034 Barcelona, Spain \\ adriana.hansberg@upc.edu
}

Submitted: Dec 14, 2011; Accepted: Jul 23, 2012; Published: Aug 9, 2012

Mathematics Subject Classifications: 05C55, 05 C65

\begin{abstract}
The family $D(k, m)$ of graphs having an orientation such that for every vertex $v \in V(G)$ either (outdegree) $\operatorname{deg}^{+}(v) \leqslant k$ or (indegree) $\operatorname{deg}^{-}(v) \leqslant m$ have been investigated recently in several papers because of the role $D(k, m)$ plays in the efforts to estimate the maximum directed cut in digraphs and the minimum cover of digraphs by directed cuts. Results concerning the chromatic number of graphs in the family $D(k, m)$ have been obtained via the notion of $d$-degeneracy of graphs. In this paper we consider a far reaching generalization of the family $D(k, m)$, in a complementary form, into the context of $r$-uniform hypergraphs, using a generalization of Hakimi's theorem to $r$-uniform hypergraphs and by showing some tight connections with the well known Ramsey numbers for hypergraphs.
\end{abstract}

Keywords: oriented hypergraphs, Ramsey $p$-chromatic number; $d$-degenerate hypergraph; Ramsey numbers; chromatic number

\section{Introduction}

The family $D(k, m)$ of graphs having an orientation such that for every vertex $v \in V(G)$ either (outdegree) $\operatorname{deg}^{+}(v) \leqslant k$ or (indegree) $\operatorname{deg}^{-}(v) \leqslant m$ have been investigated recently in several papers because of the role $D(k, m)$ plays in the efforts to estimate the maximum directed cut in digraphs and the minimum cover of digraphs by directed cuts. Results concerning the chromatic number of graphs in the family $D(k, m)$ have been obtained

*Supported by a Juan de la Cierva Postdoctoral Fellowship; Research also supported by the Ministry of Education and Science, Spain, and the European Regional Development Fund (ERDF) under project MTM2011-28800-C02-02; and under the Catalonian Government project 1298 SGR2009. 
via the notion of $d$-degeneracy of graphs (see $[2,5,7,16,18]$ ). A main tool in obtaining results on $D(k, m)$ is the following well known theorem of Hakimi. For a graph $G$, the maximum average degree of $G$ is defined as $\operatorname{Mad}(G)=\max \frac{2|E(F)|}{|V(F)|}$, where the maximum is taken over all non-empty subgraphs $F$ of $G$.

Theorem 1 (Hakimi, [15]). Let $G$ be a graph. Then $G$ has an orientation such that the maximum outdegree of $G$ is at most $k$ if and only if $\operatorname{Mad}(G) \leqslant 2 k$.

In this paper we consider a far reaching generalization of the family $D(k, m)$, in a complementary form, into the context of $r$-uniform hypergraphs. To present a sample of our results we need the following definitions.

1) Let $H$ be an $r$-uniform hypergraph. An orientation of $H$ associates with each edge an ordering of its vertices; an edge of size $r$ can be ordered in $r$ ! ways. Let $D(H)$ denote an orientation of $H$. Let $P_{1}, P_{2}, \ldots, P_{\left(\begin{array}{c}r \\ p\end{array}\right)}$ be the $p$-sets of $\{1, \ldots, r\}$, representing the possible sets of positions that gets a $p$-set contained in an edge $E$ under orientation $D(H)$. For a subset $A \subseteq V(H)$ with $|A|=p$, let $d_{i}(A)$ denote the number of edges in $D(H)$ in which the set of positions occupied by $A$ on the given orientation of $H$ is precisely $P_{i}$. When $A=\{v\}$, we simply write $d_{i}(v)$ for $d_{i}(\{v\}), 1 \leqslant i \leqslant r$. We define the degree vector of length $\left(\begin{array}{l}r \\ p\end{array}\right)$ as the vector with coordinates $d_{i}(A), 1 \leqslant i \leqslant\left(\begin{array}{l}r \\ p\end{array}\right)$, representing the number of occurrences of $A$ in each of the $\left(\begin{array}{l}r \\ p\end{array}\right)$ positions in which $A$ appears in the oriented edges of $D(H)$. Further, $\Delta_{i}(D)=\max _{v \in V} \operatorname{deg}_{i}(v)$ is the maximum among the $i$-th coordinates of the degree vectors of the vertices of $H$ under orientation $D$.

2) Define $f(D(H), p, k)$ as the number of $p$-sets $A \subseteq V(H)$ with $d_{i}(A) \geqslant k$ for all $p$-sets $P_{i} \subseteq\{1, \ldots, r\}, 1 \leqslant i \leqslant\left(\begin{array}{l}r \\ p\end{array}\right)$, and denote $f(H, p, k)$ the minimum of $f(D(H), p, k)$ over all orientations $D(H)$ of $H$. We also use $f(n, r, p, k)$ in case that $H=H(n, r)=\left(K_{n}\right)^{r}$ is the complete $r$-uniform hypergraph. For graphs, as $p=1$, we use the shorter notation $f(G, k)$ for $f(G, 1, k)$. Define further $f(r, p, k)$ as the minimum $n$ such that in every orientation of the complete $r$-uniform hypergraph $H(n, r)$ there is a $p$-set with all coordinates at least $k$. Thus $f(r, p, k)=\min \{n: f(n, r, p, k)>0\}$.

3) Let $H$ be an $r$-uniform hypergraph. Suppose we color the $p$-sets of $V(H)$ by some colors. An edge $E$ is $p$-monochromatic if all its $p$-sets receive the same color. The Ramsey $p$-chromatic number $\chi_{R}(H, p)$ is the minimum number of colors used in coloring the $p$ sets of $V(H)$ such that no edge is $p$-monochromatic. Note that $\chi_{R}(H, 1)$ is the traditional chromatic number $\chi(H)$ of $H$. Denote by $\chi_{R}(n, r, p)$ the Ramsey $p$-chromatic number of $H(n, r)$, that is, $\chi_{R}(n, r, p)$ is the minimum integer $t$ such that the $p$-sets of $H(n, r)$ can be colored by $t$ colors without a $p$-monochromatic edge (a monochromatic copy of $H(r, p))$. Hence, in a sense, $\chi_{R}(n, r, p)$ is the inverse of the Ramsey numbers. For example, $\chi_{R}(n, 3,2)=2$ for $3 \leqslant n \leqslant 5$, but $\chi_{R}(6,3,2)=3$ since $R\left(K_{3}, K_{3}\right)=6$, then $\chi_{R}(n, 3,2)=3$ for $6 \leqslant n \leqslant 16$ but $\chi_{R}(17,3,2)=4$ since $R\left(K_{3}, K_{3}, K_{3}\right)=17$. Since

$$
(*) \quad c_{1}(321)^{k / 5} \leqslant R\left(K_{3}: k \text { colors }\right) \leqslant 3 k !
$$

we get $c_{2} \log n \geqslant \chi_{R}(n, 3,2) \geqslant c_{3} \log n / \log \log n$. The left bound (*) was given by Exoo in [11], while the right bound $(\dagger)$ is from Chung and Grinstead [9]. 
4) Let $B(H, p)$ be a largest family of $p$-sets of $V(H)$ that can be colored using at most $\left(\begin{array}{l}r \\ p\end{array}\right)$ colors such that no edge of $H$ with all its $p$-sets in $B(H, p)$ is $p$-monochromatic. Let $|B(H, p)|=b(H, p)$. Thus if $\chi_{R}(H, p) \leqslant\left(\begin{array}{l}r \\ p\end{array}\right)$ then $b(H, p)=\left(\begin{array}{l}n \\ p\end{array}\right)$.

5) We say that an $r$-uniform hypergraph $H$ is $r$-partite if $V(H)$ can be partitioned into at most $r$ independent sets. Note that for an $r$-uniform hypergraph $H, b(H, 1)$ is the cardinality of the largest induced $r$-partite subhypergraph of $H$.

6) For an $r$-uniform hypergraph $H$, define $\operatorname{Mad}(H)=\max \{\operatorname{re}(F) /|F|: \emptyset \neq F \subseteq V(H)\}$.

7) For an $r$-uniform hypergraph $H$, define $M(H, k)=\max \left\{\left|A_{1} \cup A_{2} \cup \ldots \cup A_{r}\right|\right\}$, where the maximum is taken among all mutually vertex disjoint subsets $A_{i}$ such that $\operatorname{Mad}\left(A_{i}\right) \leqslant r k$, for $i=1,2, \ldots, r$.

8) Recall that a hypergraph $H$ is $d$-degenerate if in every induced subhypergraph $F$ of $H$ (including $H$ itself) there is a vertex whose degree in $F$ is at most $d$. A classical result of Szekeres and Wilf [22] states that, if a graph $G$ is $d$-degenerate, then $\chi(G) \leqslant d+1$. This theorem extends easily to hypergraphs, namely if $H$ is a $d$-degenerate hypergraph then $\chi(H) \leqslant d+1$.

9) We shall now complete the notation used in this paper. Given a hypergraph $H$ with vertex set $V(H)$ and edge set $E(H)$, the number edges of $H$ is denoted by $e(H)$. With $\operatorname{deg}(v)$ we denote the degree of $v \in V$, i.e. the number of edges containing $v$. We denote by $\delta(H)$ the minimum among all degrees of the vertices of $H$. Let $\bar{d}(H)=\frac{1}{n} \sum_{v \in V} \operatorname{deg}(v)$ be the average degree of $H$. We will deal with $r$-uniform hypergraphs, i.e. hypergraphs having every edge of size $r$. We denote with $H(n, r)$ the complete $r$-uniform hypergraph on $n$ vertices. For a subset $A \subseteq V$ of the vertex set of $H$, the induced subhpergraph $H[A]$ of $H$ by $A$ is the hyperpgraph with vertex set $A$ and all edges $E \in E(H)$ such that $E \subseteq A$. Moreover, for $v \in A, \operatorname{deg}(v: A)$ stands for the degree of $v$ in $H[A]$. We call a subset $S \subseteq V$ independent if $|S \cap E| \leqslant r-1$ for every edge $E \in E(H)$ and with $\alpha(H)$ we denote the maximum cardinality of an independent set of $H$.

All these concepts are valid also for graphs $(r=2)$, where we write $G$ instead of $H$. Finally, we denote by $K_{n}$ the complete graph on $n$ vertices and by $K_{a, b}$ is the complete bipartite graph with partite sets of cardinality $a$ and $b$.

The following is a sample of our main results:

1) Let $H$ be an $r$-uniform hypergraph. Then there is an orientation of $H$ such that for every vertex $v \in V(H)$, the (outdegree) $d_{1}(v) \leqslant k$ if and only if $\operatorname{Mad}(H) \leqslant r k$.

$2)$ Let $H$ be an $r$-uniform hypergraph on $n$ vertices. Then $f(H, 1, k)=n-M(H, k-1)$.

3) Let $H$ be an $r$-uniform hypergraph. Then $f(H, 1, k) \geqslant \chi(H)-r(r(k-1)+1)$.

4) $f(n, r, 1, k)=\max \{n-r t, 0\}$, where $t$ is the maximum integer such that $\left(\begin{array}{c}t-1 \\ r-1\end{array}\right) \leqslant(k-1) r$.

5) $f(H, p, 1) \geqslant\left(\begin{array}{l}n \\ p\end{array}\right)-b(H, p)$ where equality holds for $p=1$ and $p=r-1$.

6) $f(r, r-1,1)=R(H(r, r-1), r)$, the Ramsey number of $H(r, r-1)$ using $r$ colors. In particular $f(3,2,1)=17, f(4,3,1) \leqslant 15202$ (see [20])

7) Let $H(n . r)$ be the complete $r$-uniform hypergraph on $n$ vertices. Suppose $r>p \geqslant 1$, $t \geqslant p, k \geqslant 1$, and $n \geqslant N(r, p, t, k)$. Then in every orientation $D(H)$ there is a $t$-set $B$ of $V(H)$ all its $\left(\begin{array}{l}t \\ p\end{array}\right) p$-sets having degree-vector with all coordinates at least $k$. 
8) We determine $f(G, k)$ for several families of graphs including complete $t$-partite graphs, maximal outerplanar graphs and maximal planar graphs.

The paper is organized as follows.

\section{Contents}

1 Introduction $\quad 1$

2 Generalization of Hakimi's Theorem to hypergraphs 4

3 Bounds on $f(H, 1, k)$ and $f(H, p, 1) \quad 5$

3.1 Bounds using the generalization of Hakimi's Theorem to hypergraphs . . . 5

3.2 Lower bound using the chromatic number . . . . . . . . . . . . . . . . 9

3.3 Concrete results for families of graphs . . . . . . . . . . . . . . . . . 10

4 Ramsey type theorem for $f(H, p, k) \quad 17$

4.1 Bounds using the notion of $\mathrm{b}(H, p) \ldots \ldots \ldots \ldots$. . . . . . . . 17

4.2 Bounds using Ramsey numbers . . . . . . . . . . . . . . . . . . . . . . 19

$\begin{array}{lll}5 & \text { Open problems } & 21\end{array}$

\section{Generalization of Hakimi's Theorem to hypergraphs}

To prove the generalization of Hakimi's theorem to $r$-uniform hypergraphs, we need the following result:

Lemma 2 (Frank, Király, Király [12]). Let $H(V, E)$ be a hypergraph and let $f: V \rightarrow \mathbb{Z}^{+}$ be a mapping of the vertex set $V$ of $H$ into the set of non-negative integers. Then there is an orientation $D(H)$ of $H$ such that $\operatorname{deg}_{1}(v)=f(v)$ for every $v \in V$ if and only if

(i) $\sum_{v \in V} f(v)=e(H)$ and

(ii) $\sum_{v \in F} f(v) \geqslant e(F)$ for every $F \subseteq V$.

Lemma 3. Let $H=(V, E)$ be a hypergraph and let $f: V \rightarrow \mathbb{Z}^{+}$be a mapping of the vertex set $V$ of $H$ into the set of non-negative integers. Suppose that, for every $F \subseteq V$, $\sum_{v \in F} f(v) \geqslant e(F)$. Then there is an orientation $D(H)$ of $H$ such that $\operatorname{deg}_{1}(v) \leqslant f(v)$ for every $v \in V$.

Proof. Let $g: V \rightarrow \mathbb{Z}^{+}$be a mapping such that

(i) for every $F \subseteq V, \sum_{v \in F} g(v) \geqslant e(F)$;

(ii) $g(v) \leqslant f(v)$ for every $v \in V$;

(iii) $\sum_{v \in V} g(v)$ is the minimum among all functions that satisfy (i) and (ii). 
We will show that $\sum_{v \in V} g(v)=e(H)$ and then, by Lemma 2, there is an orientation $D(H)$ of $H$ for which $\operatorname{deg}_{1}(v)=g(v) \leqslant f(v)$ for every $v \in V$ and we are done. Let $X \subseteq V$ be a set with maximum cardinality for which $\sum_{v \in X} g(v)=e(X)$. Possibly $X$ is the empty set. If $X=V$ we are done, so we assume $|X|<|V|$. If $w$ is a vertex in $V \backslash X$ for which $g(w)=0$, then by maximality of $|X|$ we obtain

$$
\sum_{v \in X \cup\{w\}} g(v)>e(X \cup\{w\}) \geqslant e(X)=\sum_{v \in X} g(v)+0=\sum_{v \in X} g(v)+g(w)=\sum_{v \in X \cup\{w\}} g(v),
$$

a contradiction. Hence $V \backslash X$ contains no vertex $w$ with $g(w)=0$. Let $z$ be in $V \backslash X$ such that $g(z)>0$ and define $h: V \rightarrow \mathbb{Z}^{+}$such that $h(v)=g(v)$ for $v \in V \backslash\{z\}$ and $h(z)=g(z)-1$. Suppose now that there is a subset $F \subseteq V$ such that $\sum_{v \in F} h(v)<e(F)$. Then $z \in F$ and $e(F) \leqslant \sum_{v \in F} g(v)=1+\sum_{v \in F} h(v)<1+e(F)$, which implies that $\sum_{v \in F} g(v)=e(F)$. But, by the maximality of $|X|$ and since $F$ is not contained in $X$ as $z \in F$ and $z \in V \backslash X$, we have that

$$
\sum_{v \in F \cup X} g(v)>e(F \cup X) \geqslant e(F)+e(X)=\sum_{v \in F} g(v)+\sum_{v \in X} g(v) \geqslant \sum_{v \in F \cup X} g(v),
$$

again a contradiction. It follows that $\sum_{v \in F} h(v) \geqslant e(F)$ for all $F \subseteq V$ and thus $h$ is a function satisfying (i). Since evidently $h(v) \leqslant g(v) \leqslant f(v)$ for all $v \in V, h$ also satisfies (ii). However $\sum_{v \in V} h(v)<\sum_{v \in V} g(v)$, which contradicts the minimality of $g$. Hence $|X|=|V|$ and we are done.

Theorem 4. JLet $H$ be an $r$-uniform hypergraph and $k \geqslant 0$ an integer. Then there is an orientation of $H$ such that, for all $v \in V, \operatorname{deg}_{1}(v) \leqslant k$ if and only if $\operatorname{Mad}(H) \leqslant r k$.

Proof. We proceed proving first the necessity. Let $D$ be an orientation of $H$ such that, for all $v \in V, \operatorname{deg}_{1}(v) \leqslant k$ and let $X$ be a subset of $V \operatorname{such}$ that $\operatorname{Mad}(H)=r e(X) /|X|$. Let $D^{*}$ be the induced orientation from $D$ on $X$. Then $e(X)=\sum_{v \in X} \operatorname{deg}_{1}(v: X) \leqslant$ $\sum_{v \in X} \operatorname{deg}_{1}(v) \leqslant k|X|$. Hence multiplying by $r$ and dividing by $|X|$, we obtain $\operatorname{Mad}(H)=$ $r e(X) /|X| \leqslant r k$.

For the sufficiency, let $f(v)=k$ for every $v \in V$. Since $\operatorname{Mad}(H) \leqslant r k$, we get $r e(F) /|F| \leqslant r k$ for every $F \subseteq V$ and $e(F) \leqslant k|F|=\sum_{v \in F} f(v)$. Hence by Lemma 3 there is an orientation of $H$ such that $\operatorname{deg}_{1}(v) \leqslant k$ for every $v \in V$.

\section{Bounds on $f(H, 1, k)$ and $f(H, p, 1)$}

\subsection{Bounds using the generalization of Hakimi's Theorem to hypergraphs}

The next theorem reveals a basic relation between $f(H, 1, k)$ and $M(H, k-1)$.

Theorem 5. Let $H$ be an r-uniform hypergraph on $n$ vertices. Then $f(H, 1, k)=n-$ $M(H, k-1)$. 
Proof. For the upper bound, let $A_{1}, \ldots, A_{r}$ be $r$ mutually vertex disjoint sets realizing $M(H, k-1)$ and let $B=V \backslash \bigcup_{i=1}^{r} A_{i}$. Since $\operatorname{Mad}\left(A_{i}\right) \leqslant r(k-1)$, then by Theorem 4, we can orient the edges in $A_{i}$ such that the $\operatorname{deg}_{i}(v) \leqslant k-1$ for all $v \in A_{i}$. Further, orient the edges in $B$ arbitrarily. Now we have to take care for edges which are not contained in some $A_{i}$ or $B$ without violating $\operatorname{deg}_{i} \leqslant k-1$ in $A_{i}, i=1, \ldots, r$. Let $E$ be an edge such that $E \backslash A_{i} \neq \emptyset$, and such that $E \backslash B \neq \emptyset$ (i.e. such that it is neither contained in any $A_{i}$ nor in $\left.B\right)$. Set $a_{i}=\left|E \cap A_{i}\right|$ and $b=|E \cap B|$. Clearly, $b+\sum_{1 \leqslant i \leqslant r} a_{i}=r$ and there are at least two positive summands and all $a_{i} \leqslant r-1$. Define a bipartite graph $T$ with one side the vertex set $X=\{1, \ldots, r\}$ the other side the vertex set $E$ and the edges such that, for $v \in E$ and $i \in X, v i$ is an edge if and only if $v \notin A_{i}$. If we show that a perfect matching exists in this bipartite graph $T$, then this perfect matching supplies an order

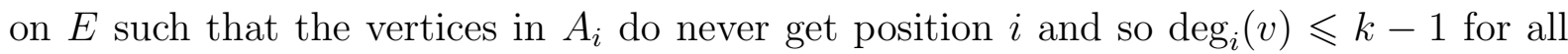
$v \in A_{i}$. For a subset $Q$ of $E$ consider the following cases. If $|Q|=r$, then $Q=E$ and $Q$ contains vertices either from some $A_{i}$ and $A_{j}$ or from some $A_{i}$ and $B$ and in both cases $|N(Q)|=r=|Q|$. If otherwise $|Q| \leqslant r-1$, then, as every vertex in $E$ has at least $r-1$ neighbors in $X$, it follows clearly that $|N(Q)| \geqslant r-1 \geqslant|Q|$ and we are done by Hall's Theorem.

Hence there is an order on $E$ that does not violate $\operatorname{deg}_{i}(v) \leqslant k-1$ for each $v \in A_{i}$, $i=1, \ldots, r$ and we are done. Hence there are at least $M(H, k-1)$ vertices in which, for some $i=1, \ldots, r, \operatorname{deg}_{i}(v) \leqslant k-1$, proving $n-M(H, k-1) \geqslant f(H, 1, k)$.

For the lower bound, let $D$ be an orientation of $H$ that realizes $f(H, 1, k)$. Let $A_{1}$ be the set of all vertices $v$ with $\operatorname{deg}_{1}(v) \leqslant k-1$ and, for $i=2, \ldots, r$, let $A_{i}$ be the set of vertices $v$ not in $\bigcup_{j=1}^{i-1} A_{j}$ with $\operatorname{deg}_{i}(v) \leqslant k-1$. Consider the induced orientation $D_{i}$ on $H\left[A_{i}\right]$. Since $\Delta_{i}\left(D_{i}\right) \leqslant k-1$, then by Theorem 4 , it follows that $\operatorname{Mad}\left(A_{i}\right) \leqslant r(k-1)$. Since the sets $A_{i}, 1 \leqslant i \leqslant r$, are pairwise vertex disjoint, it follows that $\left|\bigcup_{i=1}^{r} A_{i}\right| \leqslant M(H, k-1)$. Hence $f(H, 1, k)=n-\left|\bigcup_{i=1}^{r} A_{i}\right| \geqslant n-M(G, k-1)$.

Combining the upper and the lower bound we obtain $f(H, 1, k)=n-M(H, k-1)$.

Let $G$ be a graph. Note that, since $f(G, 1)=n-M(G, 0)$, to determine if $f(G, 1) \leqslant$ $n-k$ is equivalent to prove that $M(G, 0) \geqslant k$. As $M(G, 0)$ is precisely the maximum order of an induced bipartite subgraph of $G$ and this problem is well-known to be NP-complete [17], the problem to determine if $f(G, 1) \leqslant n-k$ is clearly NP-complete, too.

Theorem 6. Let $t$ be the maximum integer such that $\left(\begin{array}{c}t-1 \\ r-1\end{array}\right) \leqslant(k-1) r$. Then

$$
f(n, r, 1, k)=\max \{n-r t, 0\} .
$$

In particular, $f\left(K_{n}, k\right)=f(n, 2,1, k)=n-4 k+2, f(n, 3,1, k)=n-3\left\lfloor\frac{\sqrt{24 k-23}+3}{2}\right\rfloor$ and $f(n, r, 1, k)<n-e^{-1}(r-1) r^{\frac{r}{r-1}}(k-1)^{\frac{1}{r-1}}$.

Proof. Let $H=H(n, r)$. Let $t$ be the maximum integer such that $\left(\begin{array}{c}t-1 \\ r-1\end{array}\right) \leqslant(k-1) r$. If $A \subseteq V(H)$ is a set such that $\operatorname{Mad}(A) \leqslant r(k-1)$, then $\frac{r}{|A|}\left(\begin{array}{c}|A| \\ r\end{array}\right) \leqslant r(k-1)$, which is equivalent to $\left(\begin{array}{c}|A|-1 \\ r-1\end{array}\right) \leqslant r(k-1)$. Hence $|A| \leqslant t$ and the maximum cardinality of such a 
set $A$ with $\operatorname{Mad}(A) \leqslant r(k-1)$ is precisely $t$. Thus $M(H(n, r), k-1)=\min \{r t, n\}$. Now Theorem 5 yields $f(n, r, 1, k)=\max \{n-r t, 0\}$.

When $r=2, t$ is easily computed to $2 k-1$, while when $r=3$, it is not difficult to check that $t=\left\lfloor\frac{\sqrt{24 k-23}+3}{2}\right\rfloor$. Finally, using $r(k-1)<\left(\begin{array}{c}t \\ r-1\end{array}\right) \leqslant\left(\frac{t e}{r-1}\right)^{r-1}$, we obtain that $t>(r-1) e^{-1}(r(k-1))^{\frac{1}{r-1}}$, implying that $f(n, r, 1, k)=n-r t<n-e^{-1}(r-1) r^{\frac{r}{r-1}}(k-$ $1)^{\frac{1}{r-1}}$.

Corollary 7. Let $G$ be a graph on $n$ vertices with minimum degree $\delta(G) \geqslant(t-1) \frac{n}{t}$. Then $f(G, k) \geqslant(t-4 k+2)\left\lfloor\frac{n}{t}\right\rfloor$.

Proof. Since $\delta(G) \geqslant(t-1) \frac{n}{t}$ it follows from the Hajnal-Szemeredi Theorem [14] that $G$ has $\left\lfloor\frac{n}{t}\right\rfloor$ vertex-disjoint copies of $K_{t}$. Each copy of $K_{t}$ supplies, by Theorem 6, at least $t-4 k+2$ vertices with indegree and outdegree at least $k$. Hence we have at least $(t-4 k+2)\left\lfloor\frac{n}{t}\right\rfloor$ vertices with indegree and outdegree at least $k$.

Theorem 5 allows us to deduce a Turan's type result for the maximum number of edges in an $r$-uniform hypergraph $H$ with $f(H, 1, k)=0$.

Theorem 8. Let $H$ be an $r$-uniform hypergraph with $f(H, 1, k)=0$. Then

$$
e(H) \leqslant\left(\begin{array}{c}
n \\
r
\end{array}\right)-r\left(\begin{array}{c}
n / r \\
r
\end{array}\right)+(k-1) n
$$

and this bound is sharp for $n>(k-1) r^{2 r-1}$ when $r^{2}$ divides $n$.

Proof. Since $f(H, 1, k)=0$, Theorem 5 implies that $M(H, k-1)=n$. Let now $A_{1} \cup A_{2} \cup$ $\ldots \cup A_{r}$ be $r$ vertex disjoint sets with $\operatorname{Mad}\left(A_{i}\right) \leqslant r(k-1), 1 \leqslant i \leqslant r$, realizing $M(H, k-1)$. Then $\left|A_{1} \cup A_{2} \cup \ldots \cup A_{r}\right|=n$. Then, by convexity, we have that $\sum_{i=1}^{r}\left(\begin{array}{c}\left|A_{i}\right| \\ r\end{array}\right) \geqslant r\left(\begin{array}{c}n / r \\ r\end{array}\right)$. Moreover, as $\frac{r e\left(A_{i}\right)}{\left|A_{i}\right|} \leqslant \operatorname{Mad}\left(A_{i}\right) \leqslant r(k-1)$, it follows that $e\left(A_{i}\right) \leqslant(k-1)\left|A_{i}\right|$ for all $i=1,2, \ldots, r$. Hence the number of edges of $H$ is at most

$$
e(H) \leqslant\left(\begin{array}{c}
n \\
r
\end{array}\right)-\sum_{i=1}^{r}\left(\begin{array}{c}
\left|A_{i}\right| \\
r
\end{array}\right)+\sum_{i=1}^{r} e\left(A_{i}\right) \leqslant\left(\begin{array}{c}
n \\
r
\end{array}\right)-r\left(\begin{array}{c}
n / r \\
r
\end{array}\right)+(k-1) n .
$$

To see the sharpness, let $r^{2}$ divide $n$ and take $\left|A_{i}\right|=n / r$ for $, i=1,2, \ldots, r$. Then $r$ divides $\left|A_{i}\right|$. By the well-known Theorem of Baranyai [6], the $r$-uniform hypergraph induced on $A_{i}$ has a 1-factorization. In particular, take precisely $r(k-1) 1$-factors. Each 1-factor contributes with $\left|A_{i}\right| / r$ edges and so we obtain exactly $r(k-1)\left|A_{i}\right| / r=(k-1)\left|A_{i}\right|$ edges in each $A_{i}$. Altogether, we obtain $(k-1) n$ edges and thus we have equality in the inequality given above. Taking $r(k-1)$ 1-factors is possible if $r(k-1) \leqslant \frac{e\left(A_{i}\right)}{\left|A_{i}\right| / r}=\left(\begin{array}{c}n / r \\ r\end{array}\right) \frac{r^{2}}{n}$ (which gives the total number of 1-factors). Hence $r(k-1) \leqslant \frac{r^{2}}{n}\left(\begin{array}{c}n / r \\ r\end{array}\right)$, which gives $n(k-1)<r\left(\begin{array}{c}n / r \\ r\end{array}\right)<r\left(\frac{e n}{r^{2}}\right)^{r}$. Taking logarithm we obtain $\log (n)+\log (k-1)<\log r+$ $r \log \left(\frac{e n}{r^{2}}\right)=\log r+r \log (e n)-2 r \log r=r+r \log n-(2 r-1) \log r$. Hence $\log (k-1)<$ $(r-1) \log n+r-(2 r-1) \log r$. Rearranging we get $\frac{\log (k-1)-r+(2 r-1) \log r}{r-1}<\log n$. This is indeed fulfilled when $n>(k-1) r^{2 r-1}$, since then $\log n>\log (k-1) r^{2 r-1}=\log (k-1)+$ $\log r^{2 r-1}>\frac{\log (k-1)-r+(2 r-1) \log r}{r-1}$. 

lemmas.

In order to get more information from Theorem 5 we need the following technical

Lemma 9. Let $H$ be an $r$-uniform hypergraph on $n$ vertices with $\operatorname{Mad}(H) \leqslant k$, where $k$ is a non-negative integer. Then:

(1) For every subhypergraph $F$ of $H, \operatorname{Mad}(F) \leqslant k$.

(2) $H$ is $k$-degenerate.

(3) $H$ is $(k+1)$-colorable.

(4) $\alpha(H) \geqslant n /(k+1)$.

Proof. (1) This is evident from the definition of $\operatorname{Mad}(H)$.

(2) $H$ is $k$-degenerate since in every subhypergraph $F$ of $H$ (including $\mathrm{H}) \delta(F) \leqslant \bar{d}(F) \leqslant$ $\operatorname{Mad}(F) \leqslant \operatorname{Mad}(H) \leqslant k$.

(3) By the Szekeres-Wilf Theorem for hypergraphs (see [22], the same proof as for graphs) if $H$ is $d$-degenerate, then $\chi(H) \leqslant d+1$. Hence in our case $\chi(H) \leqslant k+1$.

(4) Observe that $\alpha(H) \geqslant \frac{n}{\chi(H)} \geqslant \frac{n}{k+1}$.

Lemma 10. Let $k \geqslant 0$ be an integer. The following assertions hold.

(1) If $H$ is a $k$-degenerate $r$-uniform hypergraph, then $\operatorname{Mad}(H) \leqslant r k$.

(2) Suppose $H$ is an $(r(k+1)-1)$-degenerate hypergraph. Then $V(H)$ can be partitioned into $r$ vertex disjoint subsets $V(H)=\bigcup_{i=1}^{r} A_{i}$ such that the induced subhypergraph on $A_{i}$ is $k$-degenerate for each $1 \leqslant i \leqslant r$.

Proof. (1) Since $H$ is $k$-degenerate, so does every subhypergraph $F$ of $H$. Hence for every subhypergraph $F$ we have by induction $e(F) \leqslant k|F|$ and hence $r e(F) /|F| \leqslant r k$ and $\operatorname{Mad}(H) \leqslant r k$.

(2) Suppose first that $|V(H)| \leqslant r$. Clearly $H$ has at most one edge and is 1-degenerate and as $1 \leqslant r(0+1)-1=r-1, H$ is $(r-1)$-degenerate and we can partition $V(H)$ into singletons which are 0-degenerate. Suppose we have proven the result for $(r(k+1)-1)$-degenerate hypergraphs of order $n$. Let now $H$ be an at most $(r(k+1)-1)$-degenerate $r$-uniform hypergraph of order $n+1$. By $(r(k+1)-1)$-degeneracy and Lemma $9(2)$, there is a vertex $v$ with $\operatorname{deg}(v) \leqslant r(k+1)-1$ and such that $H^{*}=H-v$ is also $(r(k+1)-1)$-degenerate. By induction, $V\left(H^{*}\right)$ can be partitioned into $r$ vertex disjoint subsets $A_{i}, 1 \leqslant i \leqslant r$, all of them inducing $k$-degenerate subhypergraphs. Since $\operatorname{deg}(v) \leqslant r(k+1)-1$, there is at least one $A_{i}$ with at most $k$ edges in $A_{i} \cup\{v\}$. But then $A_{i} \cup\{v\}$ is again a $k$-degenerate subhypergraph.

For the following, define $\beta_{d}(H)$ as the maximum cardinality $|F|$ over all subsets $F \subseteq$ $V(H)$ such that the induced subhypergraph on $F$ is $d$-degenerate. Note that $\beta_{0}(H)=$ $\alpha(H)$.

Theorem 11. Let $H$ be an r-uniform hypergraph on $n$ vertices and $G$ a graph. Then the following assertions hold. 
(1) $f(H, 1, k)=n-M(H, k-1) \geqslant n-\alpha(H) r(r k-r+1)$

(2) $n-\beta_{r k-1}(H) \geqslant f(H, 1, k) \geqslant n-r \beta_{r(k-1)}(H)$.

(3) If $G$ has average degree $\bar{d} \geqslant 4 k-2$, then $f(G, k) \leqslant \frac{\bar{d}-2 k+1}{\bar{d}+1} n$.

Proof. (1) Let $A_{i}, i=1, \ldots, r$ be $r$ vertex disjoint classes such that $\operatorname{Mad}\left(A_{i}\right) \leqslant r(k-1)$ and $\left|\bigcup_{i=1}^{r} A_{i}\right|=M(H, k-1)$. By Lemma $9(4), A_{i}$ contains an independent set $X_{i}$ of cardinality $\left|X_{i}\right| \geqslant \frac{\left|A_{i}\right|}{r(k-1)+1}=\frac{\left|A_{i}\right|}{r k-r+1}$. Clearly,

$$
r \alpha(H) \geqslant \sum_{i=1}^{r}\left|X_{i}\right| \geqslant \sum_{i=1}^{r} \frac{\left|A_{i}\right|}{r k-r+1}=\frac{M(H, k-1)}{r k-r+1} .
$$

Hence, $\alpha(H) r(r k-r+1) \geqslant M(H, k-1)$. From this we get

$$
f(H, 1, k)=n-M(H, k-1) \geqslant n-\alpha(H) r(r k-r+1)
$$

(2) We have to show that $\beta_{r k-1}(H) \leqslant M(H, k-1) \leqslant r \beta_{r(k-1)}(H)$.

(i) Let $F \subseteq V(H)$ be a set such that its induced subhypergraph of $H$ is $(r k-1)$-degenerate and such that $|F|=\beta_{r k-1}(H)$. By Lemma 10(2), $V(H)$ can be partitioned into $r$ vertex disjoint subsets $A_{i}$ each of them inducing a $(k-1)$-degenerate subhypergraph. By Lemma 10(1), $\operatorname{Mad}\left(A_{i}\right) \leqslant r(k-1)$ and hence $|F|=\left|\bigcup_{i-1}^{r} A_{i}\right| \leqslant M(H, k-1)$.

(ii) Let $A_{i}, i=1, \ldots, r$ be $r$ vertex disjoint classes such that $\operatorname{Mad}\left(A_{i}\right) \leqslant r(k-1)$ and $\bigcup_{i=1}^{r} A_{i} \mid=M(H, k-1)$. By Lemma $9(2), A_{i}$ is $r(k-1)$-degenerate and hence $\left|A_{i}\right| \leqslant$ $\beta_{r(k-1)}(H)$ and $M(G, k-1)=\left|\cup A_{i}\right| \leqslant r \beta_{r(k-1)}(H)$. Hence with (i) and (ii) and Theorem 5 , we have

$$
n-r \beta_{r(k-1)}(H) \leqslant f(H, 1, k)=n-M(H, k-1) \leqslant n-\beta_{r k-1}(H)
$$

and we are done.

(3) By a result given in [3], if $\bar{d}(G) \geqslant 2 d$, then $\beta_{d}(G) \geqslant \frac{d+1}{\bar{d}+1} n$. In our case set $d=2 k-1$, so if $\bar{d} \geqslant 2(2 k-1)=4 k-2$, then $\beta_{2 k-1}(G) \geqslant 2 k n /(\bar{d}+1)$. Hence, by Theorem $11(2)$,

$$
f(G, k) \leqslant n-\beta_{2 k-1}(G) \leqslant n-\frac{2 k n}{\bar{d}+1}=\frac{\bar{d}-2 k+1}{\bar{d}+1} n .
$$

\subsection{Lower bound using the chromatic number}

Theorem 12 is a generalization for $r$-uniform hypergraphs of the case $r=2$ proved in [2].

Theorem 12. Let $H$ be an $r$-uniform hypergraph on $n$ vertices. Then $f(H, 1, k) \geqslant \chi(H)-$ $r(r(k-1)+1)$. This bound is sharp in case that $r=2$. 
Proof. Let $H$ be a $r$-uniform hypergraph with chromatic number $\chi(H)>r(r(k-1)+1)$, otherwise there is nothing to prove. We will show first that in any orientation $D(H)$ of $H$ there is at least one vertex $v$ with $\operatorname{deg}_{i}(v) \geqslant k$ for $i=1,2, \ldots, r$. Suppose there is an orientation $D=D(H)$ of $H$ such that for every vertex $x \in V$ there is an index $j$ such that $\operatorname{deg}_{j}(x)<k$. Let $A_{1}$ be the set of vertices $u$ with $\operatorname{deg}_{1}(u)<k$. Define recursively $A_{j}$ as the set of vertices in $u \in V \backslash \cup_{i=1}^{j-1} A_{i}$ with $\operatorname{deg}_{j}(u)<k$, where some $A_{i}$ are possibly empty. If $A_{i}$ is not empty, then for all vertices $v \in A_{i}$ we have that $\operatorname{deg}_{i}(v)<k$, in the induced orientation of the $r$-uniform subhypergraph induced by $A_{i}$ still all vertices $v \in A_{i}$ have $\operatorname{deg}_{i}(v)<k$. By Theorem 4, this is possible if and only if $\operatorname{Mad}\left(A_{i}\right) \leqslant r(k-1)$. By Lemma 9 (3), it follows that $H\left[A_{i}\right]$ is $r(k-1)+1$-colorable. Hence we can take for each nonempty $A_{i} r(k-1)+1$ different and new colors and we obtain $\chi(H) \leqslant r(r(k-1)+1)$, which is a contradiction.

So, every orientation $D(H)$ of $H$ contains a vertex $v$ with $\operatorname{deg}_{i}(v) \geqslant k$ for all $i=$ $1,2, \ldots, r$. Now let us prove the theorem by induction on $\chi(H)$. The case $k=1$ is easy, so we assume that $k \geqslant 2$. For $\chi(H)=r(r(k-1)+1)+1$, as we already showed, there is indeed one vertex, say $u$, with $\operatorname{deg}_{i}(u) \geqslant k$ for all $i=1,2, \ldots, r$. Now assume we have proved the theorem for $\chi(H)=t \geqslant r(r(k-1)+1)+1$. Let now $H$ have chromatic number $\chi(H)=t+1$ and let $D(H)$ be any orientation of $H$. Consider $H^{*}=H-u$, where evidently $\chi\left(H^{*}\right) \geqslant t$. If $\chi\left(H^{*}\right)=t$, then by the induction hypothesis in every orientation of $H^{*}$, including the orientation induced by $D(H)$, there are at least $t-r(r(k-1)+1)$ vertices with all coordinates of their degree vector at least $k$. Then, together with $u$, there are at least $t-r(r(k-1)+1)+1=(t+1)-r(r(k-1)+1)$ vertices with all coordinates at least $k$ and we are done. Hence suppose that $\chi\left(H^{*}\right)=t+1$. Then again there is a vertex $u^{\prime} \in V\left(H^{*}\right)$ with all its coordinates at least $k$ and we may consider $H^{* *}=H^{*}-u^{\prime}$ and repeat. Hence we have proved that there are at least $\chi(H)-r(r(k-1)+1)$ vertices with all coordinates at least $k$ and we obtain $f(H, 1, k) \geqslant \chi(H)-r(r(k-1)+1)$.

To prove the sharpness for $r=2$, consider the complete graph on $n$ vertices $K_{n}$, where clearly $\chi\left(K_{n}\right)=n$ and, by Theorem $6, f\left(K_{n}, k\right)=n-4 k+2$. Hence $f\left(K_{n}, k\right)=$ $n-4 k+2=\chi(H)-2(2(k-1)+1)$.

\subsection{Concrete results for families of graphs}

For a graph $G$, define $\alpha_{2}(G)=\max |A \cup B|$, where the maximum is taken over all vertex disjoint independent sets $A$ and $B$. Clearly, $\alpha_{2}(G)=M(G, 0)$. Observe that trivially $2 \alpha(G) \geqslant \alpha_{2}(G) \geqslant \alpha(G)$ with the lower bound attained if and only if $G=\overline{K_{n}}$. With this definition we have the following corollary to Theorem 11.

Corollary 13. Let $G$ be a graph on $n$ vertices. Then the following holds.

(1) $n-\alpha(G) \geqslant f(G, 1) \geqslant n-2 \alpha(G)$ and both bounds are sharp.

(2) If there are two vertex-disjoint independent sets of cardinality $\alpha(G)$, then $f(G, 1)=$ $n-2 \alpha(G)$.

(3) If $\chi(G)=t$, then $f(G, 1) \leqslant\left\lfloor\frac{(t-2)}{t} n\right\rfloor$ and this bound is sharp. 
Proof. (1) Observe that trivially $2 \alpha(G) \geqslant \alpha_{2}(G) \geqslant \alpha(G)$ and since $\alpha_{2}(G)=M(G, 0)$, substituting in Theorem 11, we get the desired result. Another proof of the lower bound is as follows. The Gallai-Milgram Theorem (see [13]) states that every oriented graph $G$ with independence number $\alpha(G)$ has a vertex partition into $\alpha(G)$ oriented paths. So let $P_{1}, P_{2}, \ldots, P_{\alpha(G)}$ be such a partition. Let $P_{1}, \ldots, P_{t}$ be the paths consisting of a unique vertex and $P_{t+1}, \ldots, P_{\alpha(G)}$ the paths consisting each of at least two vertices. Clearly, each of the paths $P_{t+1}, \ldots, P_{\alpha(G)}$ contribute with $\left|P_{i}\right|-2$ vertices whose coordinates are at least 1 (those vertices except the head and the tail of the path). Hence, in every orientation $D$ of $G$, there are at least $\sum_{i=t+1}^{\alpha(G)}\left|P_{i}\right|-2$ vertices with coordinates at least 1 . However,

$$
\begin{aligned}
\sum_{i=t+1}^{\alpha(G)}\left|P_{i}\right|-2 & =\left|P_{1}\right|+\ldots+\left|P_{t}\right|-t+\sum_{i=t+1}^{\alpha(G)}\left|P_{i}\right|-2 \\
& =\sum_{i=1}^{\alpha(G)}\left|P_{i}\right|-2(\alpha(G)-t)-t \\
& =n-2 \alpha(G)+t \geqslant n-2 \alpha(G) .
\end{aligned}
$$

So, in every orientation of $G$ there are at least $n-2 \alpha(G)$ vertices with indegree and outdegree at least 1 .

Note that the upper bound is attained if and only if $G=\overline{K_{n}}$. Otherwise, if $G$ has size at least one, then $n-\alpha(G)-1 \geqslant f(G, 1)$. Further, the lower bound is attained for example by the graph $G=t K_{m}$ where $\alpha(G)=t$ and $f(G, 1)=t(m-2)$, since by Theorem $6 f\left(K_{m}, 1\right)=m-2$. Hence $f(G, 1)=t m-2 t=n-2 \alpha(G)$.

(2) In this case $M(G, 0)=2 \alpha(G)$ and the result follows.

(3) Suppose that $t \geqslant 2$, otherwise there is nothing to prove. Let $A_{1}, \ldots, A_{t}$ be the color classes such that $\left|A_{1}\right| \geqslant \ldots \geqslant\left|A_{t}\right|$. Then $\left|A_{1}\right| \geqslant \frac{n}{t}$ and $\left|A_{2}\right| \geqslant \frac{n-\left|A_{1}\right|}{t-1}$. Hence $\alpha_{2}(G) \geqslant$ $\left|A_{1} \cup A_{2}\right| \geqslant\left|A_{1}\right|+\frac{n-\left|A_{1}\right|}{t-1}=\frac{(t-2)\left|A_{1}\right|+n}{t-1}$ and then $n-\frac{(t-2)\left|A_{1}\right|+n}{t-1} \geqslant n-\alpha_{2}(G)=f(G, 1)$. This implies that $\frac{\left(n-\left|A_{1}\right|\right)(t-2)}{t-1} \geqslant f(G, 1)$ and, since $\left|A_{1}\right| \geqslant \frac{n}{t}$, we obtain

$$
\frac{(n-n / t)(t-2)}{t-1}=\frac{(t-1) n(t-2)}{(t-1) t}=\frac{n(t-2)}{t} \geqslant f(G, 1)
$$

and hence $f(G, 1) \leqslant\left\lfloor(t-2) \frac{n}{t}\right\rfloor$. Another proof of this upper bound is as follows. Let $A_{1}, \ldots, A_{t}$ be the partition of $V$ into $\chi(G)=t$ independent sets and assume $\left|A_{1}\right| \geqslant \ldots \geqslant$ $\left|A_{t}\right|$. Orient all edges from $A_{1}$ to $V \backslash A_{1}$ and all the edges into $A_{2}$ from $V \backslash A_{2}$. Clearly, the vertices in $A_{1}$ have indegree exactly 0 and the vertices in $A_{2}$ have outdegree exactly 0. Clearly, $\left|A_{1} \cup A_{2}\right| \geqslant 2 \frac{n}{t}$ and hence in the above orientation there are at most $(t-2) n / t$ vertices with indegree and outdegree at least 1 .

That this bound is sharp can be seen by the complete $t$-partite graph with all parts equal to $n / t$. Then clearly the graph has a $K_{t}$-factor containing $n / t$ vertex-disjoint copies of $K_{t}$. In every orientation of $G$ every copy of $K_{t}$ supplies $t-2$ vertices with indegree and outdegree at least 1 . Altogether we have (by vertex disjointness) at least $\lfloor(t-2) n / t\rfloor$ vertices with indegree and outdegree at least 1 . 
If $G=K_{n_{1}, n_{2}, \ldots, n_{t}}$, i.e. a a complete $t$-partite graph with partition sets $V_{i}$ of cardinality $\left|V_{i}\right|=n_{i}, 1 \leqslant i \leqslant t$, then we are able to compute $f(G, k)$. For this purpose we will first prove the following lemma.

Lemma 14. Let $G$ be a complete t-partite graph $K_{n_{1}, n_{2}, \ldots, n_{t}}$ with partition sets $V_{i}$ of cardinality $\left|V_{i}\right|=n_{i}$ for $1 \leqslant i \leqslant t, t \geqslant 2$, where $n_{1} \geqslant n_{2} \geqslant \ldots \geqslant n_{t} \geqslant 1$. Among all sets $A \subseteq \bigcup_{i=1}^{t} V_{i}=V$ of cardinality $a$, where $0<a<n=\sum_{i=1}^{t} n_{i}$, the minimum number of edges in the subgraph induced by $A$ is

$$
\min _{A \subseteq V,|A|=a} e(G[A])=\sum_{1 \leqslant i<j \leqslant q-1} n_{i} n_{j}+\left(a-\sum_{i=1}^{q-1} n_{i}\right) \sum_{i=1}^{q-1} n_{i},
$$

where $q$ is the index for which $\sum_{i=1}^{q-1} n_{i} \leqslant a<\sum_{i=1}^{q} n_{i}$.

Proof. Let $A^{*} \subseteq \bigcup_{i=1}^{t} V_{i}$ be a set with minimum number of edges $e\left(G\left[A^{*}\right]\right)$ among all subsets of cardinality $a$ and let $a_{i}=\left|V_{i} \cap A^{*}\right|$ and let $a_{i}=\left|V_{i} \cap A^{*}\right|$. Since $e\left(G\left[A^{*}\right]\right)=$ $\sum_{1 \leqslant i<j \leqslant t} a_{i} a_{j}$, we can choose a set $A^{*}$ such that $a_{1} \geqslant a_{2} \geqslant \ldots \geqslant a_{t}$. Now let $q$ be the minimum index $i$ for which $a_{i} \neq n_{i}$ and let $s$ be the maximum index $i$ for which $a_{i} \neq 0$. Evidently, $s-1 \leqslant q \leqslant s+1$. If $q=s$ or if $s+1=q$, we are done. So suppose that $q<s$. Since $a_{q} \neq n_{q}$ and $a_{s} \neq 0$, we can take vertices $x \in V_{q} \backslash A_{q}$ and $y \in A_{s}$. Define now $A_{q}^{\prime}=A_{q} \cup\{x\}$, and $A_{s}^{\prime}=A_{s} \backslash\{y\}$ and $A_{i}^{\prime}=A_{i}$ for all $i \neq q$, s. Let $A^{\prime}=\cup_{i=1}^{t} A_{i}^{\prime}$ and $a_{i}^{\prime}=\left|A_{i}\right|$. The we have

$$
\begin{aligned}
e\left(G\left[A^{\prime}\right]\right) & =\sum_{1 \leqslant i<j \leqslant t} a_{i}^{\prime} a_{j}^{\prime} \\
& =a_{q}^{\prime} \sum_{j \neq q, s} a_{j}^{\prime}+a_{q}^{\prime} a_{s}^{\prime}+a_{s}^{\prime} \sum_{j \neq q, s} a_{j}^{\prime}+\sum_{i<j, i, j \neq q, s} a_{i}^{\prime} a_{j}^{\prime} \\
& =\left(a_{q}+1\right) \sum_{j \neq q, s} a_{j}+\left(a_{q}+1\right)\left(a_{s}-1\right)+\left(a_{s}-1\right) \sum_{j \neq q, s} a_{j}+\sum_{i<j, i, j \neq q, s} a_{i} a_{j} \\
& =a_{s}+a_{q}-1+\sum_{1 \leqslant i<j \leqslant t} a_{i} a_{j}=a_{s}+a_{q}-1+e(G[A])
\end{aligned}
$$

But, since $q<s$, we have that $a_{s}-a_{q} \leqslant 0$ and hence $e\left(G\left[A^{\prime}\right]\right)<e(G[A])$, which is a contradiction to the minimality of $e\left(G\left[A^{*}\right]\right)$. Hence, $q=s$ or $s+1=q$ and thus $a_{1}=n_{i}$ for $i \leqslant q-1, a_{i}<n_{i}$ for $i=q$, and $a_{i}=0$ for $i \geqslant q+1$. This implies that

$$
e\left(G\left[A^{*}\right]\right)=\sum_{1 \leqslant i<j \leqslant q-1} n_{i} n_{j}+a_{q} \sum_{i=1}^{q-1} n_{i} .
$$

Further, as $a_{q}=a-\sum_{i=1}^{q-1} n_{i}$ and $q$ is exactly the index for which $\sum_{i=1}^{q-1} n_{i} \leqslant a<\sum_{i=1}^{q} n_{i}$, we obtain that

$$
e\left(G\left[A^{*}\right]\right)=\sum_{1 \leqslant i<j \leqslant q-1} n_{i} n_{j}+\left(a-\sum_{i=1}^{q-1} n_{i}\right) \sum_{i=1}^{q-1} n_{i},
$$

for every set $A$ of cardinality $a$. 
Corollary 15. Let $G$ be a complete $t$-partite graph $K_{n_{1}, n_{2}, \ldots, n_{t}}$ with partition sets $V_{i}$ of cardinality $\left|V_{i}\right|=n_{i}$, where $1 \leqslant i \leqslant t, t \geqslant 2$, and $n_{1} \geqslant n_{2} \geqslant \ldots \geqslant n_{t} \geqslant 1$. If $A \subseteq \bigcup_{i=1}^{t} V_{i}$ is a set of cardinality a such that $\sum_{i=1}^{q-1} n_{i} \leqslant a<\sum_{i=1}^{q} n_{i}$ for an integer $q \geqslant 1$, then, for any $\ell \in\{1,2, \ldots, q-1\}$

$$
e(G[A]) \geqslant\left(a-n_{\ell}\right) n_{\ell}
$$

Proof. Let $\ell \in\{1,2, \ldots, q-1\}$. Then using Lemma 14, we obtain

$$
\begin{aligned}
e(G[A]) & \geqslant \sum_{1 \leqslant i<j \leqslant q-1} n_{i} n_{j}+\left(a-\sum_{i=1}^{q-1} n_{i}\right) \sum_{i=1}^{q-1} n_{i} \\
& \geqslant n_{\ell} \sum_{1 \leqslant i \leqslant q-1, i \neq \ell} n_{i}+\left(a-\sum_{i=1}^{q-1} n_{i}\right) n_{\ell}=\left(a-n_{\ell}\right) n_{\ell} .
\end{aligned}
$$

Theorem 16. Let $n_{1} \geqslant n_{2} \geqslant \ldots \geqslant n_{t}$, where $t \geqslant 3, n_{1}, n_{2} \geqslant k^{2}-k+1$ and either $n_{3} \geqslant 2 k-2$ or $n_{3} \geqslant k-1$ and $n_{4} \geqslant k-1$. Then $f\left(K_{n_{1}, n_{2}, \ldots, n_{t}}, k\right)=\sum_{i=3}^{t} n_{i}-2 k+2$.

Proof. Let $V=\cup_{i=1}^{t} V_{i}$ be the partition of the vertex set of $K_{n_{1}, n_{2}, \ldots, n_{t}}$ such that $\left|V_{i}\right|=$ $n_{i}$. Let $A$ and $B$ be two vertex disjoint subsets of $V$ such that $\operatorname{Mad}(A) \leqslant 2 k-2$ and $\operatorname{Mad}(B) \leqslant 2 k-2$ and let $a_{i}=\left|A \cap V_{i}\right|, b_{i}=\left|B \cap V_{i}\right|$ for $1 \leqslant i \leqslant t$ and $|A| \geqslant|B|$. We will show that $|A \cup B| \leqslant n_{1}+n_{2}+2 k-2$.

Suppose first that $|A|=n_{1}+r$ for an integer $r>0$. Since $|A|>n_{1}$, we can set $q \geqslant 2$ in Corollary 15 and so $e(G[A]) \geqslant\left(n_{1}+r-n_{1}\right) n_{1}=r n_{1}$. Further, as $\frac{2 e(G[A])}{n_{1}+r} \leqslant \operatorname{Mad}(A) \leqslant$ $2 k-2$, we have that

$$
r n_{1} \leqslant e(G[A]) \leqslant(k-1)\left(n_{1}+r\right),
$$

which, together with $n_{2} \geqslant k^{2}-k+1$, implies that

$$
r \leqslant \frac{(k-1) n_{1}}{n_{1}-k+1}=k-1+\frac{k^{2}-2 k+1}{n_{2}-k+1} \leqslant k-1+\frac{k^{2}-2 k+1}{k^{2}-2 k+2} .
$$

Hence, $|A| \leqslant n_{1}+k-1$.

If $|B| \leqslant n_{1}+n_{2}+2 k-2-|A|$, it follows that $|A \cup B| \leqslant n_{1}+n_{2}+2 k-2$ and we are done. Hence assume that $|B|=n_{2}+s$ for an integer $s \geqslant n_{1}-|A|+2 k-1 \geqslant k$. If $b_{1} \leqslant n_{2}$, it follows by Corollary 15, setting $n_{1}^{\prime}=n_{2}, n_{i}^{\prime}=n_{i}$ for $i \geqslant 2$ and reordering the indices if necessary, that $e(G[B]) \geqslant s n_{2}$ and, analogously as above, we obtain that $s \leqslant k-1$ and thus $|A \cup B| \leqslant n_{1}+n_{2}+2 k-2$ and we are done.

Assume now for contradiction that $b_{1}=n_{2}+p$ for an integer $p$ with $s \geqslant p>0$. By Corollary 15 , setting $n_{1}^{\prime}=n_{2}+p, n_{i}^{\prime}=n_{i}$ for $i \geqslant 2$ and reordering the indices if necessary, it follows that $e(G[B]) \geqslant\left(n_{2}+p\right)(s-p)$. Since $\frac{2 e(G[B])}{n_{2}+s} \leqslant \operatorname{Mad}(B) \leqslant 2 k-2$, we have that

$$
\left(n_{2}+p\right)(s-p) \leqslant e(G[B]) \leqslant(k-1)\left(n_{2}+s\right),
$$


which, together with $n_{2} \geqslant k^{2}-k+1$, implies that

$$
\begin{aligned}
s & \leqslant \frac{n_{2}(k-1)+p\left(n_{2}+p\right)}{n_{2}+p-k+1} \\
& =\frac{\left(n_{2}+p-k+1\right)(k-1)+p\left(n_{2}+p-k+1\right)+(k-1)^{2}}{n_{2}+p-k+1} \\
& =p+k-1+\frac{(k-1)^{2}}{n_{2}+p-k+1} \\
& \leqslant p+k-1+\frac{(k-1)^{2}}{k^{2}-2 k+2+p}=p+k-1+\frac{(k-1)^{2}}{(k-1)^{2}+p+1} .
\end{aligned}
$$

Hence, $s \leqslant p+k-1$ and thus $p \geqslant s-k+1 \geqslant n_{1}-|A|+k$. Now consider $e(G[A])$. Since $a_{1} \leqslant n_{1}-b_{1} \leqslant n_{1}-n_{2}-p$, we have that $\sum_{i=2}^{t} a_{i}=|A|-a_{1} \geqslant n_{1}+k-p-a_{1} \geqslant n_{2}+k$. Thus by Corollary 15, setting $n_{1}^{\prime \prime}=n_{1}-n_{2}-p, n_{i}^{\prime \prime}=n_{i}$ for $i \geqslant 2$ and reordering the indices if necessary, it follows that $e(G[A]) \geqslant\left(|A|-n_{2}\right) n_{2}$. Note that $k^{2}-k+1 \leqslant n_{2} \leqslant n_{1}-p \leqslant$ $|A|-k$ and thus $|A| \geqslant k^{2}+1$. Define now the function $f(x)=(|A|-x) x=|A| x-x^{2}$, where $k^{2}-k+1 \leqslant x \leqslant|A|-k$. Evidently, $f(x)$ takes its minimum either when $x=k^{2}-k+1$ or when $x=|A|-k$. Since $f\left(k^{2}-k+1\right)=|A|\left(k^{2}-k+1\right)-\left(k^{2}-k+1\right)^{2}$ and $f(|A|-k)=|A| k-k^{2}$, to prove that $f\left(k^{2}-k+1\right) \geqslant f(|A|-k)$ is equivalent to

$$
|A| \geqslant \frac{\left(k^{2}-k+1\right)^{2}-k^{2}}{(k-1)^{2}}=\frac{\left((k-1)^{2}+k\right)^{2}-k^{2}}{(k-1)^{2}}=\frac{(k-1)^{4}+2 k(k-1)^{2}}{(k-1)^{2}}=k^{2}+1,
$$

which is certainly true. Hence

$$
e(G[A]) \geqslant f(|A|-k)=(|A|-k) k \geqslant|A|(k-1)+|A|-k^{2} \geqslant|A|(k-1)+1,
$$

which contradicts the assumption that $\frac{2 e(G[A])}{|A|} \leqslant \operatorname{Mad}(A) \leqslant 2 k-2$. Thus $b_{1}>n_{2}$ is not possible.

Altogether it follows that $M(G, k-1) \leqslant n_{1}+n_{2}+2 k-2$. To see that this bound is sharp, take $A=V_{1} \cup A^{\prime}$ and $B=V_{2} \cup B^{\prime}$, where $A^{\prime}$ and $B^{\prime}$ are disjoint sets such that $\left|A^{\prime}\right|=\left|B^{\prime}\right|=k-1$ and either $A^{\prime} \cup B^{\prime} \subseteq V_{3}$ in case that $n_{3} \geqslant 2 k-2$ or $A^{\prime} \subseteq V_{3}$ and $B^{\prime} \subseteq V_{4}$ in case that $2 k-2>n_{3} \geqslant k-1$ and $n_{4} \geqslant k-1$. Observe that $e(G[A])=n_{1}(k-1)$ and $e(G[B])=n_{2}(k-1)$ and thus $\operatorname{Mad}(A), \operatorname{Mad}(B) \leqslant k-1$ and, since $|A \cup B|=n_{1}+n_{2}+2 k-2$, it realizes the bound.

Hence we have proven that $M(G, k-1)=n_{1}+n_{2}+2 k-2$ and thus, with Theorem 5 , we obtain $f(G, k)=n-n_{1}-n_{2}-2 k+2=\sum_{i=3}^{t} n_{i}-2 k+2$.

A perfect graph $G$ is defined as a graph such that any of its induced subgraphs $G^{*}$, including $G$ itself, has $\chi\left(G^{*}\right)=\omega\left(G^{*}\right)$, where $\omega\left(G^{*}\right)$ stands for the clique number of $G^{*}$. Let us denote by $h\left(G, K_{3}\right)$ the cardinality of a minimum set of vertices $T$ which hits all triangles of $G$, i.e. such that every triangle contained in $G$ has at least one vertex in $T$. More information about these "triangle-hitting sets" can be found under the concepts of "clique covering" or "transversals in hypergraphs", see [1, 4, 10, 23]. Similarly, let $h(G$, odd $)$ be the cardinality of a minimum set of vertices hitting all odd cycles of $G$. Now we can state the following theorem. 
Theorem 17. If $G$ is a graph, then $f(G, 1)=h(G$, odd $) \geqslant h\left(G, K_{3}\right)$. Moreover, if $G$ is perfect, then $f(G, 1)=h\left(G, K_{3}\right)$.

Proof. Let $D$ be an orientation of $G$ realizing $f(G, 1)$ and let $S$ be the set of vertices $v$ having $\operatorname{deg}^{+}(v) \geqslant 1$ and $\operatorname{deg}^{-}(v) \geqslant 1$ under orientation $D$. As evidently every odd cycle of $G$ has to have at least one vertex in $S$ and every vertex in $S$ has to be on an odd cycle, $f(G, 1)=|S|=h(G$, odd $) \geqslant h\left(G, K_{3}\right)$.

Let now $G$ be perfect and let $T$ be a minimum set hitting all triangles of $G$. Then the graph $G^{*}=G-T$ is triangle-free and, as $G$ is perfect, $G^{*}$ is perfect as well and thus $\chi\left(G^{*}\right)=\omega\left(G^{*}\right) \leqslant 2$. Hence $G^{*}$ is bipartite and we obtain, by Theorem 5 , that $n-f(G, 1)=M(G, 0) \geqslant\left|V\left(G^{*}\right)\right|=|V \backslash T|=n-h\left(G, K_{3}\right)$. With the inequality proven above for general graphs, it follows $f(G, 1)=h\left(G, K_{3}\right)$.

A planar graph is called maximal planar, for short $M P$, if the addition of any edge would destroy that property. A maximal outerplanar graph, abbreviated $M O P$, is a triangulation of the polygon. By the Four Color Theorem, every MP graph is 4-colorable. Also, it is well-known that every MOP graph is 3-colorable. Moreover, every MP graph on $n \geqslant 3$ vertices has exactly $3 n-6$ edges, while every triangle-free planar graph on $n \geqslant 3$ vertices has at most $2 n-4$ edges.

Theorem 18. Let $G$ be a graph.

(1) If $G$ is a $M P$ on $n \geqslant 4$ vertices, then $2 \leqslant f(G, 1) \leqslant \frac{n}{2}$ and both bounds are sharp.

(2) If $G$ is a MOP on $n \geqslant 3$, then $1 \leqslant f(G, 1) \leqslant \frac{n}{3}$ and both bounds are sharp.

Proof. (1) Since there are MP graphs $G$ with $\chi(G)=3$, using the chromatic lower bound, we get only $f(G, 1) \geqslant 1$, which is exact for $n=3$. For $n \geqslant 4$, assume there is an MP graph $G$ with $f(G, 1)=1$. Then by Theorem 17 there is a vertex $v$ hitting al triangles of $G$ and thus $G^{*}=G-v$ is a triangle-free planar graph on $n-1 \geqslant 3$ vertices. Therefore, $e\left(G^{*}\right) \leqslant 2(n-1)-4=2 n-6$. But now it follows that $n-1 \geqslant \operatorname{deg}(v)=$ $e(G)-e\left(G^{*}\right) \geqslant 3 n-6-(2 n-6)=n$, which is a contradiction. Therefore, $f(G, 1) \geqslant 2$. To see the sharpness, consider the graph consisting of two adjacent vertices $u$ and $v$ and $n-2$ vertices forming a path $x_{1} x_{2} \ldots x_{n-2}$ such that both $u$ and $v$ are adjacent to every vertex on the path. Now orient the edges from $x_{i}$ out, when $i=0(\bmod 2)$, and in, when $i=1(\bmod 2)$, and lastly orient $u$ to $v$. Then $u$ and $v$ are the only vertices with indegree and outdegree at least 1 .

As every planar graph is 4-colorable, by Corollary 13(3) we have $f(G, 1) \leqslant\left\lfloor\frac{(t-2) n}{t}\right\rfloor \leqslant$ $\frac{n}{2}$, where $\chi(G)=t \leqslant 4$. To see the sharpness, take $n / 4$ vertex disjoint $K_{4}$ 's and join them by edges to a MP graph $G$. For this graph, every $K_{4}$ contributes with two vertices of indegree and outdegree at least 1 and hence $f(G, 1) \geqslant 2 n / 4=n / 2$.

(2) Let $G$ be a MOP graph. Since $\chi(G)=3$, Theorem 12 implies $f(G, 1) \geqslant \chi(G)-2=$ 1 and hence the lower bound follows. This can be realized by a graph $G$ consisting of a cycle $C_{n}$ and such that one of its vertices, say $z$, is adjacent to all other vertices. Orient the edges of the cycle in such a way that every vertex but possibly $z$ (when $n$ is odd) has either indegree or outdegree 0 . The remaining edges are oriented either to or from $z$ in 
such a way that the indegree or the outdegree of the other vertices of the cycle remains being 0 . Thus $z$ is the only vertex having $\operatorname{deg}^{+}(z)>0$ and $\operatorname{deg}^{-}(z)>0$ and hence $f(G, 1)=1$.

For the upper bound, since $\chi(G)=3$, we have $f(G, 1) \leqslant \frac{n}{3}$ by Corollary $13(3)$. To see the sharpness, take $n / 3$ vertex disjoint $K_{3}$ and complete it by adding edges to a maximal outerplanar graph $G$. As $G$ contains $n / 3$ disjoint $K_{3}$ 's, we must have $f(G, 1) \geqslant n / 3$ and thus $f(G, 1)=n / 3$ must hold. Thus the upper bound is also sharp.

Theorem 19. The following assertions hold:

(1) There exist positive constants $c_{1}(k)$ and $c_{2}(k)$ such that

$$
2 n-c_{1}(k) \log (n) \leqslant \max \{f(G, k)+f(\bar{G}, k):|V(G)|=n\} \leqslant 2 n-c_{2}(k) \log n .
$$

(2) $f(G, 1)+f(\bar{G}, 1) \geqslant n-4$ and this bound is sharp.

(3) $n-16 k+12 \leqslant \min \{f(G, k)+f(\bar{G}, k):|V(G)|=n\} \leqslant n-8 k+4$.

Proof. (1) By Corollary 13 (1), for any graph $G$ on $n$ vertices, $n-\alpha(G) \geqslant f(G, 1) \geqslant$ $n-2 \alpha(G)$ for any $n$-vetex graph $G$. Since by the Ramsey-Theorem $\max \{\alpha(G), \alpha(\bar{G})\} \geqslant$ $c \log n$ and thus $\alpha(G)+\alpha(\bar{G}) \geqslant c \log n$ for a constant $c>0$, we obtain $f(G, 1)+f(\bar{G}, 1) \leqslant$ $n-\alpha(G)+n-\alpha(\bar{G}) \leqslant 2 n-c \log n$. Further, the Ramsey-Theorem guarantees also the existence of a graph $G$ and a constant $c$ for which $\alpha(G) \sim \alpha(\bar{G}) \sim c \log n$. Hence for such graphs, $f(G, 1)+f(\bar{G}, 1) \geqslant n-2 \alpha(G)+n-2 \alpha(\bar{G}) \geqslant 2 n-4 c \log n$. Hence we have that $2 n-4 c \log n \leqslant \max \{f(G, 1)+f(\bar{G}, 1):|V(G)|=n\} \leqslant 2 n-c \log n$, which proves the theorem for $k=1$.

Since $f(G, k) \leqslant f(G, k-1)$, we also obtain for the Ramsey-graphs given above that $f(G, k)+f(\bar{G}, k) \leqslant f(G, 1)+f(\bar{G}, 1) \leqslant 2 n-c \log n$ and we can take $c_{2}(k)=c$. By Theorem 11, we have that $f(G, k) \geqslant n-2 \beta_{2(k-1)}(G)$, where $\beta_{2(k-1)}(G)$ is the cardinality of a maximum set of vertices of $G$ whose induced graph is $2(k-1)$-degenerate. Observe that for the above Ramsey-graphs, if $F$ is the largest induced 2(k-1)-degenerate graph of $G$, then $c \log n \geqslant \alpha(G) \geqslant \alpha(F) \geqslant \frac{|F|}{2 k-1}=\frac{\beta_{2(k-1)}(G)}{2 k-1}$ and hence $\beta_{2(k-1)}(G) \leqslant(2 k-1) c \log n$. The same holds for $\bar{G}$, namely $\beta_{2(k-1)}(\bar{G}) \leqslant(2 k-1) c \log n$. Hence the above Ramseygraphs have $f(G, k)+f(\bar{G}, k) \geqslant 2 n-4(2 k-1) c \log n$ and we may take $c_{1}(k)=4 c(2 k-1)$.

(2) Recall that $f(G, 1)=M(G, 0)$. Let $A$ and $B$ be two disjoint independent sets realizing $M(G, 0)$. Then in $\bar{G}, A$ and $B$ are two vertex disjoint cliques of cardinality, say, $a$ and $b$. Hence, $f(G, 1)+f(\bar{G}, 1) \geqslant n-(a+b)+(a-2)+(b-2)=n-4$ (the inequality sign is because of the possible case that $a=1$ or $b=1)$. To see that the bound can be attained, take $G=K_{a} \cup K_{b}$ and $\bar{G}=K_{a, b}$. Then $f(G, 1)=f\left(K_{a}, 1\right)+f\left(K_{b}\right)=a+b-4=n-4$, while $f(\bar{G}, 1)=f\left(K_{a, b}, 1\right)=0$. Hence $f(G, 1)+f(\bar{G}, 1)=n-4$ and the bound above is attained.

(3) Let $G$ be a graph and let $A$ and $B$ be two vertex disjoint sets realizing $M(G, 0)$. Then $\operatorname{Mad}_{G}(A) \leqslant 2(k-1)$ and $\operatorname{Mad}_{G}(B) \leqslant 2(k-1)$. Let also $X$ and $Y$ be two vertex disjoint sets realizing $M(\bar{G}, 0)$. Then $\operatorname{Mad}_{\bar{G}}(X) \leqslant 2(k-1)$ and $\operatorname{Mad}_{\bar{G}}(Y) \leqslant 2(k-1)$. Now consider any set $T \subseteq V$ such that $\operatorname{Mad}_{\bar{G}}(T) \leqslant 2(k-1)$. Then $e(\bar{G}[T]) \leqslant(k-1)|T|$ 
and hence in $G$ we have $e(G[T]) \geqslant\left(\begin{array}{c}|T| \\ 2\end{array}\right)-(k-1)|T|$. This implies that $\operatorname{Mad}_{G}(T) \geqslant$ $\frac{2 e(G[T])}{|T|}=(|T|-1)-2(k-1)$ which is greater than $2(k-1)$ when $|T|>4(k-1)+1$. Hence, as $\operatorname{Mad}_{\bar{G}}(A \cap X) \leqslant \operatorname{Mad}_{\bar{G}}(X) \leqslant 2(k-1)$, we obtain that $|A \cap X| \leqslant 4(k-1)+1$. The same happens with $B \cap X, A \cap Y$ and $B \cap Y$, and thus also $|B \cap X| \leqslant 4(k-1)+1$, $|A \cap Y| \leqslant 4(k-1)+1$ and $|B \cap Y| \leqslant 4(k-1)+1$. Now we obtain, as $A$ and $B$ are vertex disjoint and $X$ and $Y$ as well,

$$
\begin{aligned}
f(G, k)+f(\bar{G}, k) & =n-|A \cup B|+n-|X \cup Y| \\
& ==2 n-(|A \cup B \cup X \cup Y|+|A \cap X|+|A \cap Y|+|B \cap X|+|B \cap Y|) \\
& \geqslant 2 n-(n+4(4(k-1)+1))=n-16(k-1)-4=n-16 k+12 .
\end{aligned}
$$

Let now $G=K_{a} \cup K_{b}$, where $a, b \geqslant 2$ and $\bar{G}=K_{a, b}$. Then by Theorem $6 f(G, k)=$ $f\left(K_{a}, k\right)+f\left(K_{b}, k\right)=a-4 k+2+b-4 k+2=a+b-8 k+4=n-8 k+4$, while $0 \leqslant f(\bar{G}, k) \leqslant$ $f(\bar{G}, 1)=f\left(K_{a, b}, 1\right)=0$. Hence for this graph we obtain $f(G, k)+f(\bar{G}, k)=n-8 k+4$. Altogether we obtain that $n-16 k+12 \leqslant \min \{f(G, k)+f(\bar{G}, k)\} \leqslant n-8 k+4$.

\section{Ramsey type theorem for $f(H, p, k)$}

\subsection{Bounds using the notion of $\mathrm{b}(H, p)$}

Recall that, given an $r$-uniform hypergraph $H, B(H, p)$ is a largest family of $p$-sets of $V(H)$ that can be colored using at most $\left(\begin{array}{l}r \\ p\end{array}\right)$ colors such that no edge of $H$ with all its $p$-sets in $B(H, p)$ is $p$-monochromatic and $|B(H, p)|=b(H, p)$. Now we will state a bound for $f(H, p, 1)$ in terms of $b(H, p)$.

Theorem 20. Let $H$ be an $r$-uniform hypergraph and $p$ an integer with $1 \leqslant p \leqslant r-1$. Then $f(H, p, 1) \geqslant\left(\begin{array}{l}n \\ p\end{array}\right)-\mathrm{b}(H, p)$ and equality holds for $p=1$ and $p=r-1$.

Proof. Let $D$ be an orientation of the edges of $H$ that realizes $f(H, p, 1)$. Let $A_{i}$ be the set of $p$-sets $A \subseteq V$ in which the $i$-th coordinate of its degree vector is the first coordinate equal to $0,1 \leqslant i \leqslant\left(\begin{array}{l}r \\ p\end{array}\right)$. Clearly, some or even all of the $A_{i}$ can be empty and they are pairwise disjoint as no $p$-set can be in both $A_{i}$ and $A_{j}$ by definition. Since, for every edge $E$, there is a $p$-subset of $E$ on each of the possible positions $i$ for $1 \leqslant i \leqslant\left(\begin{array}{l}r \\ p\end{array}\right)$, it follows that no set $A_{i}$ can contain all $p$-sets from any edge. Thus, $\bigcup A_{i}, 1 \leqslant i \leqslant\left(\begin{array}{l}r \\ p\end{array}\right)$, is a family of $p$-sets colored by $\left(\begin{array}{l}r \\ p\end{array}\right)$ colors such that no edge is $p$-monochromatic. Hence $b(H, p) \geqslant\left(\begin{array}{l}n \\ p\end{array}\right)-f(H, p, 1)$.

We will prove now that equality holds when $p=1$ or $p=r-1$. Let $B(H, p)=\bigcup A_{i}$, $1 \leqslant i \leqslant\left(\begin{array}{l}r \\ p\end{array}\right)$, be a largest family of $p$-sets using at most $\left(\begin{array}{l}r \\ p\end{array}\right)$ colors such that no edge $E$ of $H$ with all its $p$-sets in $B(H, p)$ is $p$-monochromatic, where $A_{i}$ are the color classes. Now we have to show that every edge $E \in E(H)$ can be oriented in such a way that if $E$ contains a $p$-set $A \in A_{i}$, then the vertices of $A$ will not be placed on the set of positions corresponding to the $i$-th coordinate of the degree vector. Thus we will obtain an orientation of the edges of $H$ with at least $\mathrm{b}(H, p)$-sets with at least one zero-coordinate in its degree vector, 
showing that $f(H, p, 1) \leqslant\left(\begin{array}{l}n \\ p\end{array}\right)-b(H, p)$. Such an order is possible in case that $p=1$ or $p=r-1$.

(a) Let $p=1$. Suppose that $E=\left\{v_{1}, v_{2}, \ldots, v_{r}\right\}$ and consider all the permutations of the vertices of $E$. The probability that a vertex $v$ is placed on a forbidden position is $1 / r$ if $\{v\} \in \bigcup A_{i}$ and 0 otherwise. Hence the expected number of vertices of $E$ placed in a forbidden position is

$$
\sum_{v \in E \cap\left(\bigcup A_{i}\right)} \frac{1}{r} \leqslant 1,
$$

and equality holds when $E \subseteq \bigcup A_{i}$. So if $E$ is not contained in $\bigcup A_{i}$, the expected number of vertices placed in a forbidden position is less than 1 and thus the required order exists, namely there is an order of $E$ such that no vertex $(p=1)$ is placed in a forbidden position. Otherwise, if $E \subseteq \bigcup A_{i}$, as the $A_{i}$ 's are independent (and no edge contained in $B(H, p)$ is $p$-monochromatic), there has to be a placement of $u \in A_{i}$ and $v \in A_{j}, i \neq j$ with $u, v \in E$ such that they are placed in the $i$-th and $j$-th positions and the rest of $E$ is placed arbitrarily among the color classes. This is an ordering of $E$ in which at least two vertices are placed in forbidden positions. But, as the expected number of vertices in forbidden positions is equal to 1 , this implies that there has to be also an ordering of $E$ with no vertex placed into a forbidden position. Hence we have proved that there is an orientation of the edges such that all vertices in $A_{i}$ have a zero on its $i$-th coordinate of the degree vector. Thus there are at least $\mathrm{b}(H, 1)$ vertices whose degree vector contains a zero-coordinate, implying that $f(H, 1,1) \leqslant n-\mathrm{b}(H, 1)$, and with the inequality proved above, we obtain $f(H, 1,1)=n-\mathrm{b}(H, 1)$.

(b) Let $p=r-1$. Suppose that $E=\left\{v_{1}, v_{2}, \ldots, v_{r}\right\}$ and consider all the permutations of the vertices of $E$. The probability that an $(r-1)$-set $A \subseteq E$ is placed on a forbidden position is $\left(\begin{array}{c}r \\ r-1\end{array}\right)^{-1}=1 / r$ if $A \in \bigcup A_{i}$ and 0 otherwise. Hence the expected number of $(r-1)$-sets of $E$ placed in a forbidden position is

$$
\sum_{A \subseteq E \cap\left(\bigcup A_{i}\right),|A|=r-1} \frac{1}{r} \leqslant 1,
$$

and equality holds precisely when all $(r-1)$-subsets of $E$ are contained in $\bigcup A_{i}$. So if not all $(r-1)$-subsets of $E$ are contained in $\bigcup A_{i}$, then the expected number of $(r-1)$-subsets of $E$ placed in a forbidden position is less than 1 and hence the required order exists. Otherwise, i.e. if all $(r-1)$-subsets of $E$ are contained in $B(H, r-1)=\bigcup A_{i}$, as $E$ is not $(r-1)$-monochromatic, there are at least two $(r-1)$-subsets $X, Y \subseteq E$ in distinct color classes. Say that $X \in A_{i}$ and $Y \in A_{j}$. We want to show the existence of an orientation of $E$ such that $X$ and $Y$ are placed into the sets of positions corresponding, respectively, to the $i$-th and $j$-th coordinate of the degree vector. To do so for general $p$-sets, we need that the $p$-sets of positions $P_{i}$ and $P_{j}$ corresponding, respectively, to the $i$-th and $j$-th coordinates of the degree vector, are such that $\left|P_{i} \cap P_{j}\right|=|X \cap Y|$. This will allow us to orient $E$ such that $X$ has position set $P_{i}$ and $Y$ has position set $P_{j}$. For $p=r-1$, we have $\left|P_{i} \cap P_{j}\right|=r-2$ and $|X \cap Y|=r-2$ and such an orientation exists just by putting the vertices of $X \cap Y$ into positions $P_{i} \cap P_{j}$ and the vertex $v \in X \backslash Y$ into the 
position given by $P_{i} \backslash P_{j}$, while the vertex $u \in Y \backslash X$ into position $P_{j} \backslash P_{i}$. In this manner, we have produced an ordering of $E$ in which two $(r-1)$-sets are placed on forbidden positions. But, as the expected number of $(r-1)$-sets of $E$ in forbidden positions is equal to 1 , this implies that there has to be also an ordering of $E$ with no $(r-1)$-set placed in a forbidden position. Hence we have proved that there is an orientation of the edges such that all $(r-1)$-sets in $A_{i}$ have a zero on its $i$-th coordinate of the degree vector. Thus $f(H, r-1,1) \leqslant n-\mathrm{b}(H, r-1)$, and with the inequality proved above, we obtain $f(H, r-1,1)=n-\mathrm{b}(H, r-1)$.

Corollary 21. The following assertions hold:

(1) $f(r, r-1,1)=R(H(r, r-1), r)$, the Ramsey number of $H(r, r-1)$ using $r$ colors.

(2) $f(3,2,1)=17, f(4,3,1) \leqslant 15202$.

Proof. (1) By Theorem 20, $f(n, r, r-1,1)=\left(\begin{array}{c}n \\ r-1\end{array}\right)-\mathrm{b}(H(n, r), r-1)$. Since $\mathrm{b}(H(n, r), r-$ $1)$ is the cardinality of a largest family of $(r-1)$-sets that can be colored with at most $r$ colors such that no edge of $H(n, r)$ is $(r-1)$-monochromatic, it follows that $f(r, r-1,1)$ is the minimum $n$ such that any $r$-coloring of the $(r-1)$-sets of $V(H(n, r))$ has a $(r-1)$ monochromatic edge. Hence $f(r, r-1,1)=R(H(r, r-1), r)$.

(2) By (1), we obtain $f(3,2,1)=R(H(3,2), 3)=R\left(K_{3}, K_{3}, K_{3}\right)=17$ and $f(4,3,1)=$ $R(H(4,3), 3) \leqslant 15202$ (see $[20])$.

\subsection{Bounds using Ramsey numbers}

Theorem 22. There exists a constant $0<c(r, p, k)<1$ and a positive integer $N(r, p, k)$ such that if $H$ is an r-uniform hypergraph on $n \geqslant N(r, p, k)$ vertices and with $e(H) \geqslant$ $c(r, p, k)\left(\begin{array}{l}n \\ r\end{array}\right)$ edges, then $f(H, p, k)>0$.

We will prove the following theorem from which Theorem 22 is a corollary from Turan's theorem for hyergraphs.

Theorem 23. Let $H=H(n, r)$ be the complete $r$-uniform hypergraph on $n$ vertices and let $r>p \geqslant 1, t \geqslant p, k \geqslant 1$. Then there is an integer $N(r, p, t, k)>0$ such that if $n \geqslant N(r, p, t, k)$ then every orientation $D(H)$ has a $t$-set $B \subseteq V(H)$ all of its $p$-subsets $S \subseteq B$ having $\operatorname{deg}_{i}(S) \geqslant k$ for all $1 \leqslant i \leqslant\left(\begin{array}{c}r \\ p\end{array}\right)$.

Proof. Define a coloring of the $p$-sets of $\{1,2, \ldots, r\}$ with colors $1,2, \ldots,\left(\begin{array}{l}r \\ p\end{array}\right)+1$ the following way. If $S \subseteq\{1,2, \ldots, r\}$ is a $p$-set such that the $i$-th coordinate of the degree vector is the first to be at most $k-1$, then we assign $S$ the color $i$. If all of its coordinates are at least $k$, then $S$ gets color $\left(\begin{array}{l}r \\ p\end{array}\right)+1$ assigned. Let $q$ be the smallest integer such that $(k-1)\left(\begin{array}{l}q \\ p\end{array}\right)<\left(\begin{array}{l}q \\ r\end{array}\right)$. By the Ramsey Theorem for hypergraphs, if $n$ is sufficiently large, i.e. $n \geqslant N(r, p, q, t, k) \geqslant R(q, q, \ldots, q, t), q$ appearing $\left(\begin{array}{l}r \\ p\end{array}\right)$ times, then there is a $q$-set $Q$ with all its $p$-sets of the same color, for some color from $\left\{1,2, \ldots,\left(\begin{array}{c}r \\ p\end{array}\right)\right\}$, or there is a $t$-set $B$ all whose $p$-sets are colored with color $\left(\begin{array}{l}r \\ p\end{array}\right)+1$. If the latter case occurs, we are done. So 
suppose this is not the case and there is a $q$-set $Q$ with all its $p$-sets of the same color, say $\ell$, which means that, for every $p$-set $S$ of $Q, \operatorname{deg}_{\ell}(S)$ is the first coordinate of the degree vector being less than $k$. By double-counting the edges of the subhypergraph induced by $Q$, we obtain

$$
\left(\begin{array}{l}
q \\
r
\end{array}\right)=e(H[Q]) \leqslant \sum_{S \subseteq Q,|S|=p} \operatorname{deg}_{\ell}(S) \leqslant(k-1)\left(\begin{array}{l}
q \\
p
\end{array}\right),
$$

which is a contradiction to the choice of $q$. Hence there has to exist a $t$-set $B$ all whose $p$-sets are colored with color $\left(\begin{array}{l}r \\ p\end{array}\right)+1$, i.e. such that all their coordinates on the degree vectors are at least $k$.

Now we can give the proof of Theorem 22 .

Proof of Theorem 22. Let $q$ be the smallest integer such that $(k-1)\left(\begin{array}{l}q \\ p\end{array}\right)<\left(\begin{array}{l}q \\ r\end{array}\right)$ and let $H$ be an $r$-uniform hypergraph on $n \geqslant N(r, p, k)$ vertices, where $N(r, p, k)>R(q, q, \ldots, q)$, $q$ appearing $\left(\begin{array}{l}r \\ p\end{array}\right)$ times. Let $n^{\prime}=R(q, q, \ldots, q), q$ appearing $\left(\begin{array}{l}r \\ p\end{array}\right)$ times. By the TuránTheorem for hypergraphs, if $e(H)$ is suficently large, say $e(H)>c(r, p, k)\left(\begin{array}{l}n \\ r\end{array}\right)$ for a constant $0<c(r, p, k)<1$, then it contains a $H\left(n^{\prime}, r\right)$ as a subhypergraph. Let $D$ be an orientation of $H$ realizing $f(H, p, k)$. Suppose that in the induced orientation on $H\left(n^{\prime}, r\right)$, all $p$-sets of $\{1,2, \ldots, r\}$ have at least one coordinate of the degree vector less than $k$. Then, as in the proof of Theorem 23, we can color the $p$-sets of $H\left(n^{\prime}, r\right)$ with $\left(\begin{array}{l}r \\ p\end{array}\right)$ colors according to the first coordinate which is less than $k$. But now the Ramsey-Theorem for hypergraphs implies that there is a $q$-set $Q$ with all of its $p$-sets colored with the same color, which by the choice of $q$ is not possible. Hence there has to be a $p$-set in the subhypergraph $H\left(n^{\prime}, r\right)$ with all its coordinates at least $k$. This implies that $f(H, p, k) \geqslant f\left(H\left(n^{\prime}, r\right), p, k\right)>0$.

In the next theorem we will show that, in every orientation of $H(n, r)$, a positive fraction of all the $p$-sets must have all degree-vector coordinates at least $k$, as $n$ increases. For this purpose, we need to define the concept of packing. An $(n, m, p)$-packing $\mathcal{F}$ is a family of $m$-sets of $\{1,2, \ldots, n\}$ having the property that every of its $p$-sets is contained in at most one $F \in \mathcal{F}$. In [21], Rödl proved that there exists a family $\mathcal{F}$ with

$$
|\mathcal{F}| \geqslant\left(\begin{array}{c}
n \\
p
\end{array}\right)\left(\begin{array}{c}
m \\
p
\end{array}\right)^{-1}(1-o(1))
$$

where $p$ and $m$ are fixed and $n$ tends to infinity.

Theorem 24. For positive integers $n, r, p \leqslant r$ and $k$,

$$
f(n, r, p, k) \geqslant\left(\begin{array}{l}
n \\
p
\end{array}\right)\left(\begin{array}{c}
f(r, p, k) \\
p
\end{array}\right)^{-1}(1-o(1)),
$$

where $r, p$ and $k$ are fixed and the o(1)-term tends to zero as $n$ tends to infinity.

Proof. Setting $m=f(r, p, k)$, in every orientation of $H(m, r)$ there is a $p$-set with all its degree-vector coordinates at least $k$. Let $\mathcal{F}=\left\{F_{1}, F_{2}, \ldots, F_{t}\right\}$ be a maximum $(n, m, p)$ packing. Consider every set $F_{i}$ with all its $p$-sets as a copy of $H(m, p)$ into $H(n, p)$, 
$1 \leqslant i \leqslant t$. Thus, any two copies of $H(m, p)$ have at most $p-1$ vertices in common. Now consider an orientation of $H(n, r)$ and the induced orientation on the copies of $H(m, r)$ defined on the vertex sets $F_{1}, F_{2}, \ldots, F_{t}$. Clearly, no $p$-set belongs to two copies of $H(m, p)$ defined on the same ground set of vertices. Also, as $p \leqslant r$, no $r$-edge belongs to two copies of $H(m, r)$. Hence, in this orientation, each copy of $H(m, p)$ contributes with at least one $p$-set with all its degree-vector coordinates at least $k$. Since all these sets are distinct, it follows that $f(n, r, p, k) \geqslant t$. Then, by Rödl's Theorem, we obtain

$$
f(n, r, p, k) \geqslant t \geqslant\left(\begin{array}{l}
n \\
p
\end{array}\right)\left(\begin{array}{c}
m \\
p
\end{array}\right)^{-1}(1-o(1)),
$$

where $m$ and $p$ are fixed and $n$ grows.

\section{Open problems}

When $G$ is a maximal planar graph on $n \geqslant 4$ vertices, we have shown that $2 \leqslant f(G, 1) \leqslant$ $n / 2$, and both bounds are sharp. Since planar graphs are 5-degenerate then, by Lemma $10(2), V(G)$ can be partitioned into two vertex disjoint sets $V_{1}$ and $V_{2}$ such that their induced subgraphs are 2-degenerate. By Lemma $10(1), \operatorname{Mad}\left(V_{1}\right) \leqslant 4$ and $\operatorname{Mad}\left(V_{2}\right) \leqslant 4$ and hence $M(G, 2)=n$ and $f(G, 3)=n-M(G, 2)=0$. So every planar graph has an orientation for which every vertex $v$ has either $\operatorname{deg}^{+}(v) \leqslant 2$ or $\operatorname{deg}^{-}(v) \leqslant 2$. We are left with the problem of $f(G, 2)$ for MP graphs, which is equivalent to the following problem:

Problem 25. Does there exist an MP graph $G$ such that $V(G)$ can be partitioned into two vertex disjoint sets $A$ and $B$ such that $\operatorname{Mad}(A) \leqslant 2$ and $\operatorname{Mad}(B) \leqslant 2$ ?

There are examples of planar graphs which do not have such a partition with $\operatorname{Mad}(A)<$ 2 and $\operatorname{Mad}(B)<2$. For this, see the literature on the vertex arboricity of maximal planar graphs, for instance [8, 19, 24].

An easy upper bound for $f(G, 2)$ comes from the fact that an MP graph $G$ can be vertex-partitioned into three subsets, each of one induces a forest (i.e. a 1-degenerate subgraph). Let $V(G)=A \cup B \cup C$ be the such a partition, where $|A| \geqslant|B| \geqslant|C|$. Then $M(G, 1) \geqslant 2 n / 3$ and hence $f(G, 2)=n-M(G, 1) \leqslant n / 3$. Another observation is that if there is a planar graph $G$ on $m$ vertices with $f(G, 2)=q>0$, then we can take $r$ copies of $G$ and complete them to a MP graph $H$ on $r m$ vertices. Then each copy of $G$ gives $q$ vertices with $\operatorname{deg}^{+}(v) \geqslant 2$ and $\operatorname{deg}^{-}(v) \geqslant 2$. Hence $f(H, 2) \geqslant r q=\frac{q n}{m}$. This gives that $\max \{f(G, 2): G$ n-vertex MP graph $\} \geqslant \frac{q}{m} n$. Hence either $\max \{f(G, 2)$ : $G$ n-vertex MP graph $\}=0$ or there exists a constant $c>0$ such that $\max \{f(G, 2)$ : $G$ n-vertex MP graph $\} \geqslant c n$.

Problem 26. Prove or disprove that $\max \{f(G, 2): G$ n-vertex MP graph $\}=0$. Does every planar graph be in the class $D(1,1)$ ?

Problem 27. Find lower and upper bounds on $f(G, k)$ where $G$ belongs to the following families of graphs: claw-free graphs, $K_{1, r}$-free graphs, Line graphs, $K_{3}$-free graphs, seriesparallel graphs, $k$-trees, etc. 
Problem 28. Improve the bound $f(H, 1, k) \geqslant \chi(H)-r(r(k-1)+1)$ or show sharpness for $r>2$.

Problem 29. Improve the bounds on $f(n, r, p, k)$ given in Section 4 .

Problem 30. Is it true that $f(H, p, 1)=\left(\begin{array}{l}n \\ p\end{array}\right)-\mathrm{b}(H, p)$ for $2 \leqslant p \leqslant r$ ?

Problem 31. Can we prove at least $f(n, r, p, 1)=\left(\begin{array}{l}n \\ p\end{array}\right)-\mathrm{b}(H(n, r), p)$ for $2 \leqslant p \leqslant r$ ?

Problem 32. Is it possible to further generalize Hakimi's theorem from the case $r \geqslant 2$, $p=1$ to $r \geqslant p \geqslant 2$ ?

\section{Acknowledgements}

We would like to thank the anonymous referee for his/her very constructive suggestions and comments.

\section{References}

[1] N. Alon. Transversal numbers of uniform hypergraphs. Graphs Combin., 6(1):1-4, 1990.

[2] N. Alon, B. Bollobás, A. Gyárfás, J. Lehel, and A. Scott. Maximum directed cuts in acyclic digraphs. J. Graph Theory 55(1):1-13, 2007.

[3] N. Alon, J. Kahn, and P.D. Seymour. Large induced degenerate subgraphs. Graphs Combin. 3:203-211, 1987.

[4] T. Andreae. On the clique-transversal number of chordal graphs. Discrete Math. 191(1-3):3-11, 1998.

[5] Y. Bai, B. Li, nD S. Zhang. Covering the edges of digraphs in $D(3,3)$ and $D(4,4)$ with directed cuts. arXiv:1109.4671v1, 2011.

[6] Zs. Baranyai. On the factorization of the complete uniform hypergraph. Infinite and finite sets (Colloq., Keszthely, 1973; dedicated to P. Erdös on his 60th birthday), Vol. I. Vol. 10 of Colloq. Math. Soc. Janos Bolyai, pages 91-108. North-Holland, Amsterdam, 1975.

[7] S. Bessy, E. Birmelé, and F. Havet. Arc-chromatic number of digraphs in which every vertex has bounded outdegree or bounded indegree. J. Graph Theory 53(4):315-332, 2006.

[8] G. Chartrand, and H. V. Kronk. The point-arboricity of planar graphs. J. London Math. Soc. 44:612-616, 1969.

[9] F. R. K. Chung, and C. M. Grinstead. A survey of bounds for classical Ramsey numbers. J. Graph Theory 7(1):25-37, 1983.

[10] P. Erdős, T. Gallai, and Z. Tuza. Covering the cliques of a graph with vertices. Discrete Math. 108(1-3):279-289, 1992. 
[11] G. Exoo. A lower bound for Schur numbers and multicolor Ramsey numbers of $K_{3}$. Electron. J. Combin. 1 \#R8, 1994.

[12] A. Frank, T. Király, and Z. Király. On the orientation of graphs and hypergraphs. Discrete Appl. Math. 131(2):385-400, 2003.

[13] T. Gallai, and A. N. Milgram, Verallgemeinerung eines graphentheoretischen Satzes von Redei. Acta Sci. Math. (Szeged) 21:181-186, 1960.

[14] A. Hajnal, and E. Szemerédi. Proof of a conjecture of P. Erdős. Combinatorial theory and its applications II (Proc. Colloq., Balatonfüred, 1969), pages 601-623. North-Holland, Amsterdam, 1970.

[15] S. L. Hakimi. On the degree of the vertices of a directed graph. J. Franklin Inst. 279:290-308, 1965.

[16] J. Lehel, F. Maffray, and M. Preissmann. Maximum directed cuts in digraphs with degree restriction. J. Graph Theory 61(2):140-156, 2009.

[17] J. M. Lewis, and M. Yannakakis. The node-deletion problem for hereditary properties is NP-complete. ACM-SIGACT Symposium on the Theory of Computing (San Diego, Calif., 1978). J. Comput. System Sci. 20(2):219-230, 1980.

[18] S. Poljak, and V. Rödl. On the arc-chromatic number of a digraph. J. Combin. Theory Ser. B 31(2):190-198, 1981.

[19] A. Raspaud, and W. Wang. On the vertex-arboricity of planar graphs. European J. Combin. 29(4):1064-1075, 2008.

[20] S. P. Radziszowski. Small Ramsey numbers. Electron. J. Combin. 1: Dynamic Survey 1, 30 pp, 1994.

[21] V. Rödl. On a packing and covering problem,. European J. Combin. 6(1):69-78, 1985.

[22] G. Szekeres, and H. S. Wilf. An inequality for the chromatic number of a graph. J. Combin. Theory 4:1-3, 1968.

[23] Z. Tuza. Covering all cliques of a graph. Discrete Math. 86(1-3): 117-126, 1990.

[24] D. R. Wood. Acyclic, star and oriented colourings of graph subdivisions. Discrete Math. Theor. Comput. Sci. 7(1):37-50, 2005. 